\title{
Tumor Theranostics of Transition Metal lons Loaded Polyaminopyrrole Nanoparticles: Erratum
}

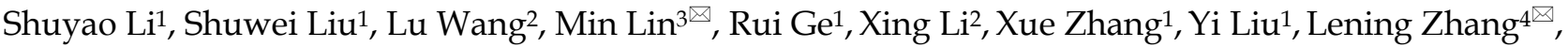 \\ Hongchen Sun ${ }^{1,2}$, Hao Zhang ${ }^{1 凶}$ and Bai Yang ${ }^{1}$ \\ 1. State Key Supramolecular Structure and Materials Laboratory, College of Chemistry, Jilin University, Changchun 130012, P. R. China; \\ 2. The Oral Pathology Department, School and Hospital attached to Stomatology, Jilin University, Changchun 130021, P. R. China; \\ 3. Collaborative Innovation Center attached to Marine Biomass Fibers, Shandong Province Materials and Textiles, Marine Biobased Materials Institute, \\ Materials Science and Engineering School, Qingdao University, Qingdao 266071, P. R. China; \\ 4. Department of Thoracic Surgery, China-Japan Union Hospital, Jilin University, Changchun 130033, P. R. China. \\ $\triangle$ Corresponding authors: hao_zhang@jlu.edu.cn (H. Zhang); hcsun@jlu.edu.cn (H.-C. Sun); linmin900401@163.com (M. Lin); zhanglening@jlu.edu.cn (L.-N. \\ Zhang).
}

(C) Ivyspring International Publisher. This is an open access article distributed under the terms of the Creative Commons Attribution License (https://creativecommons.org/ licenses/by/4.0/). See http://ivyspring.com/terms for full terms and conditions.

Published: 2022.02 .18

Corrected article: Nanotheranostics 2018; 2(3): 211-221. doi: 10.7150/ntno.25119.

In the original paper [1] on p. 218, Figure $6 \mathrm{E}$ there is error in the fluorescence image of PI and FDA co-staining cells, which arises from confusion of the images. In the revised Figure 6 shown below, the correct fluorescence images are provided. In addition, on p. 219, Figure 8D there is error in the tumors photograph of laser only group, which arises from confusion of the images. In the revised Figure 8 shown below, corrected images are provided by repeating the entire animal experiment. To ensure that the animal experiments are contrasted using the same batch of mice with Figure 8, the corresponding characterizations in Figure S9, S10 and S14 are also included with this correction. These corrections do not alter the major conclusions of this article.
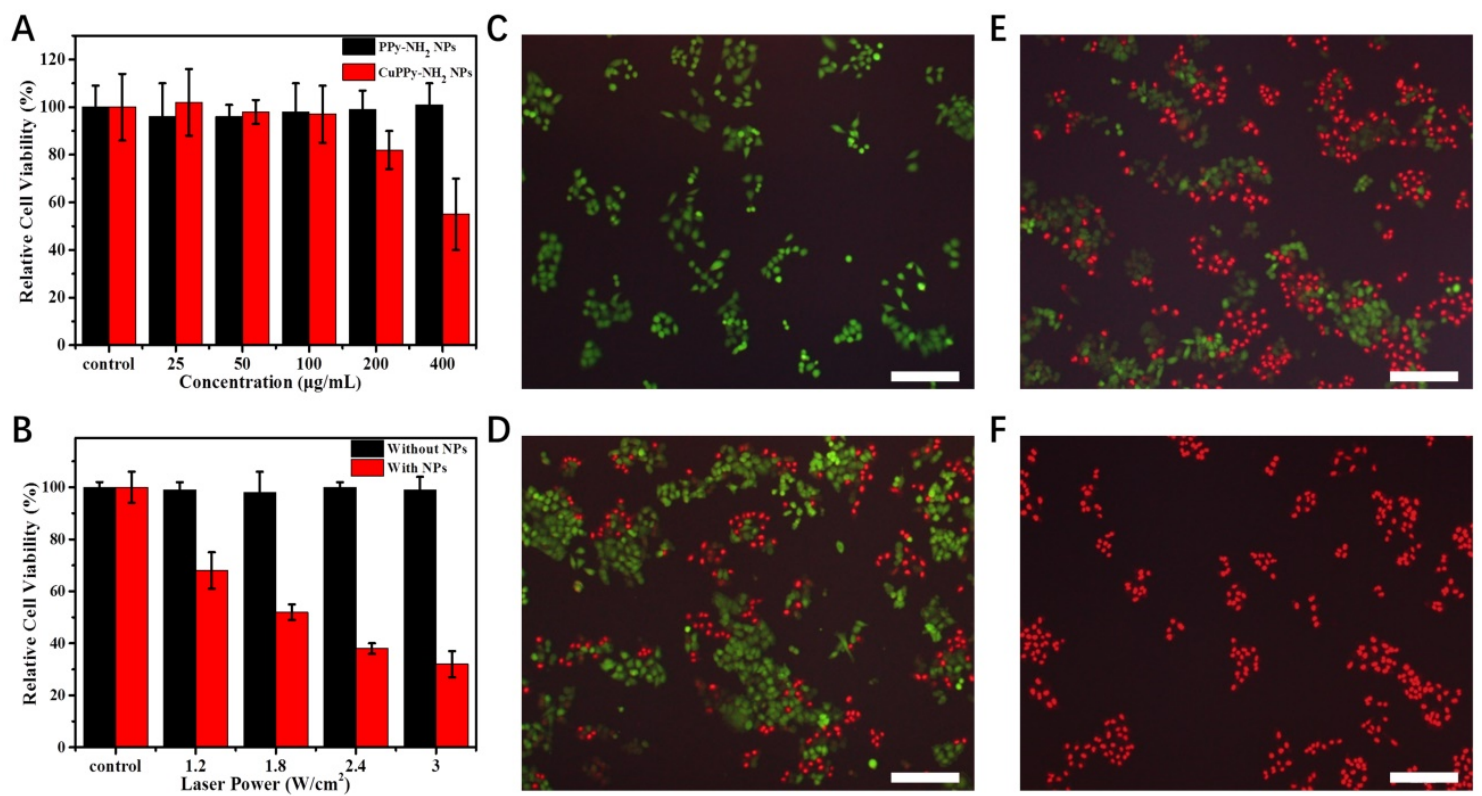

Figure 6. (A) The toxicity of $\mathrm{KB}$ cells with CuPPy-NH $\mathrm{N}_{2} \mathrm{NPs}$ and PPy- $\mathrm{NH}_{2} \mathrm{NPs}$ in different concentration. (B) $\mathrm{KB}$ cells are incubated with or without $50 \mu \mathrm{g} / \mathrm{mL} \mathrm{CuPPy}-\mathrm{NH} \mathrm{H}_{2} \mathrm{NPs}$ for $30 \mathrm{~min}$, and then they are irradiated by an $808 \mathrm{~nm}$ laser with the power density of $1.2,1.8,2.4$ and $3 \mathrm{~W} / \mathrm{cm}^{2}$ for $8 \mathrm{~min}$. Fluorescent images of PI and FDA co-staining cells after combined therapy for $0(C), 3(D), 8(E)$ and $10 \mathrm{~min}(F)$, respectively. The scale bar in (C-F) represents $200 \mu \mathrm{m}$. 
In addition, part of the description was incorrect. In "Animal experiments" (p. 213 right lines 2-10), the correct description should be " 4 weeks' old balb/c nude mice (weighing $\sim 18 \mathrm{~g}$ ) were bought from Beijing Vital River Laboratory Animal Technology Co. Ltd. The mice were used under protocols approved by Jilin University Laboratory Animal Center. After one week's feed, $2 \times 10^{6}$ of KB cells dispersed in $150 \mu \mathrm{L}$ of cell culture were injected subcutaneously into the right back leg of the mice". In "Results and Discussion" (p. 216 right line 14), the correct description should be "As exposed under laser irradiation for 0, 3, 8, $10 \mathrm{~min}$ ". These corrections do not alter the major conclusions of this article. Besides, for the laboratory animal welfare principle of "3R (Reduction, Replacement, Refinement)", we shared a blank control group with another work of our group in MRI (Figure 7G-J), which is quoted here [2].
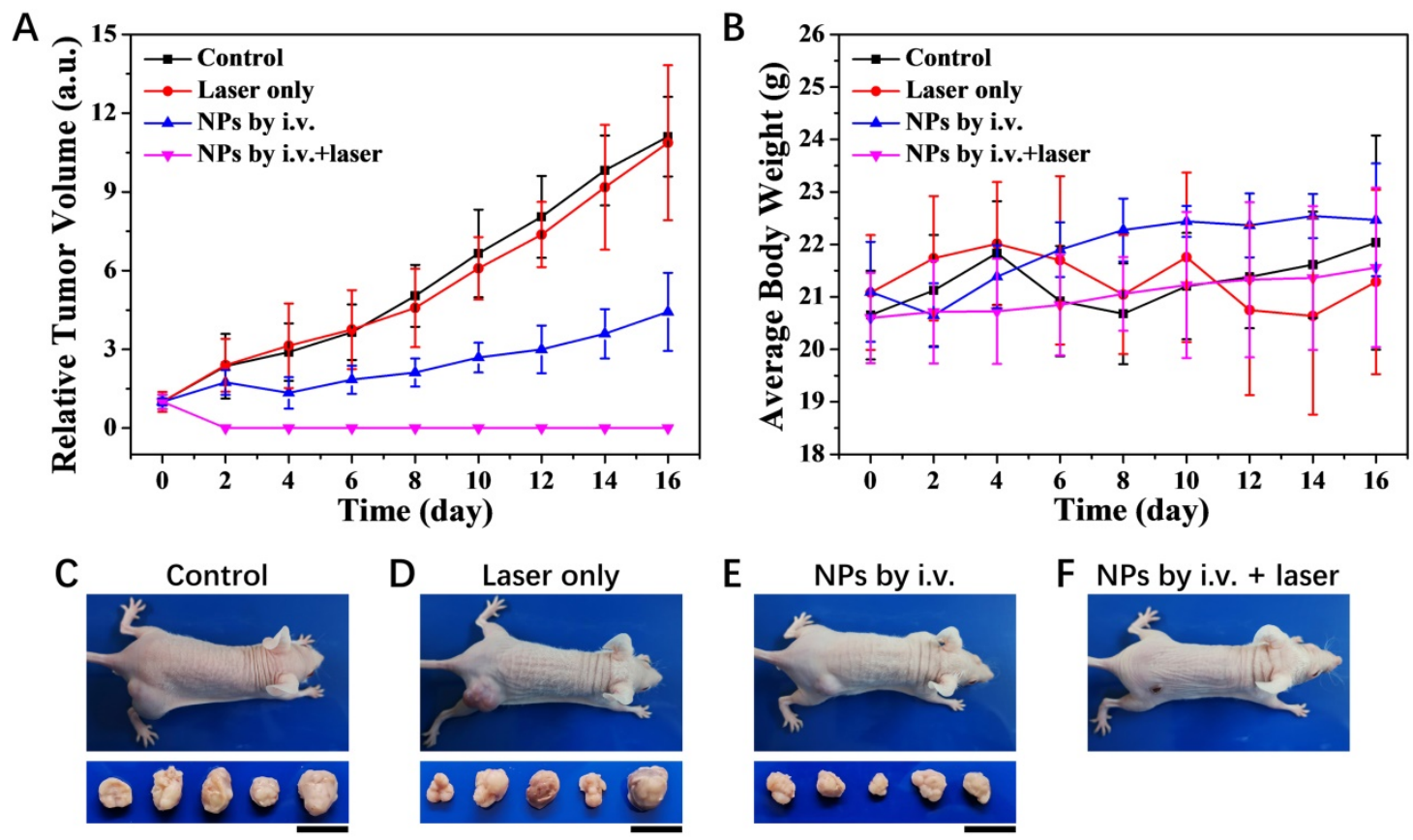

Figure 8. Photothermal therapy of KB tumors in vivo. (A) Relative tumor volume growing trend. (B) Average body weight for each group. (C-F) Photographs of typical mouse bearing tumor model and tumors taken from each group in the 16th day. The mean tumor volume in each group was $89.4,90.7,91.4$ and 92.3 mm ${ }^{3}$ at the beginning of the treatment and $992.7,986.5,404.7$ and $0 \mathrm{~mm}^{3}$ at the end of the treatment, respectively. The scale bar for tumors represents $20 \mathrm{~mm}$.
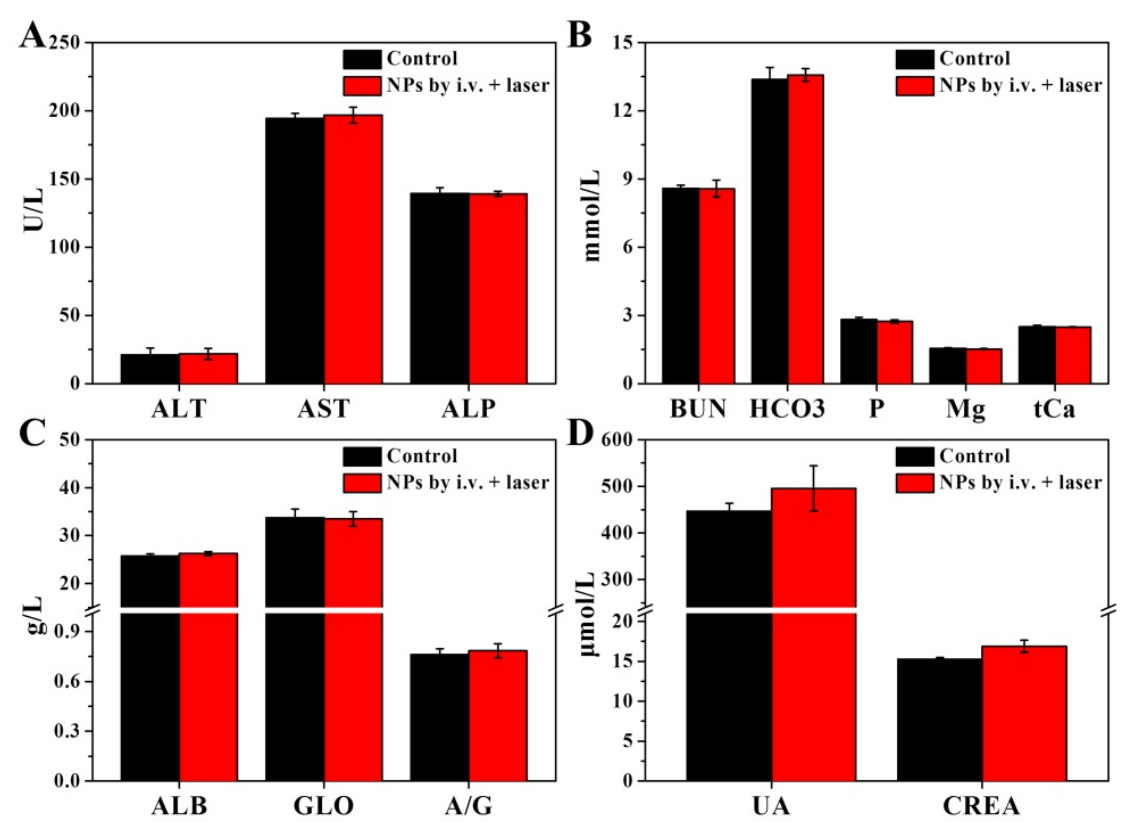

Figure A1. S9. Liver and renal functions tested $24 \mathrm{~h}$ post i.v. injection of CuPPy- $\mathrm{NH}_{2}$ NPs. All of the parameters are in normal scale comparing to the healthy control. (A) Alanine aminotransferase (ALT), aspartate transaminase (AST) and alkaline phosphatase (ALP). (B) Blood urea nitrogen (BUN), dicarbonate (HCO $\mathrm{H}_{3}$ ), serum phosphorus (P), serum magnesium (Mg) and total calcium (tCa). (C) Albumin (ALB), globulin (GLO) and the ratio of ALB to GLO (A/G). (D) Uric acid (UA) and creatinine (CREA). 
A

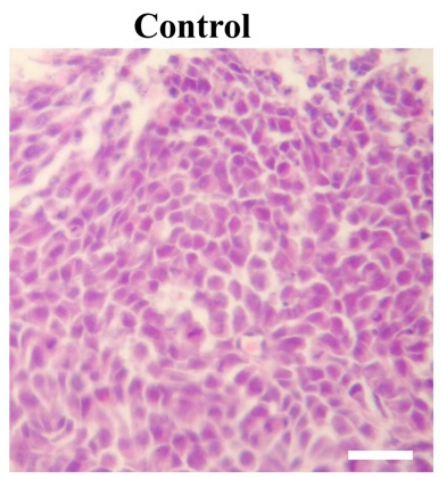

C

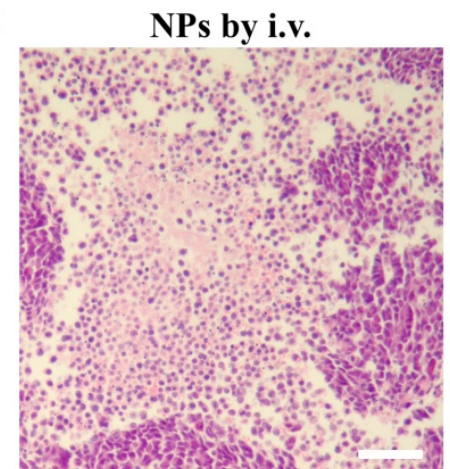

B

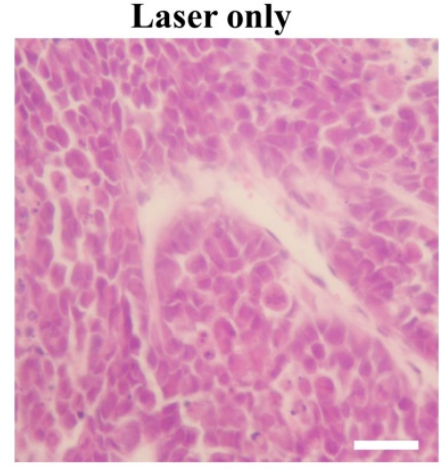

D

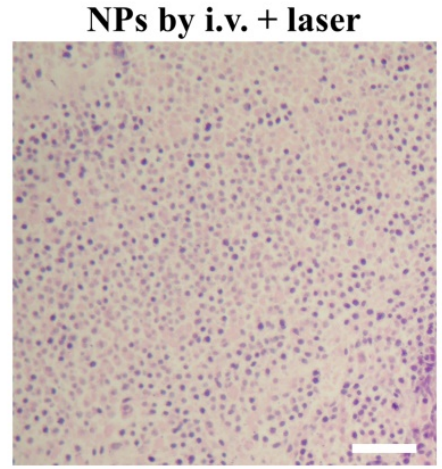

Figure A2. S10. H\&E stained tumor slices after 16 days of treatment. (A) Control group. (B) Laser only group. (C) CuPPy-NH $\mathrm{NPs}_{2}$ only group. The tumors were removed at 16 days in all three groups. (D) CuPPy- $\mathrm{NH}_{2} \mathrm{NPs}+$ laser group. In (D), after photothermal treatment with NIR laser, the tumor was removed immediately. The scale bar is 100 $\mu \mathrm{m}$

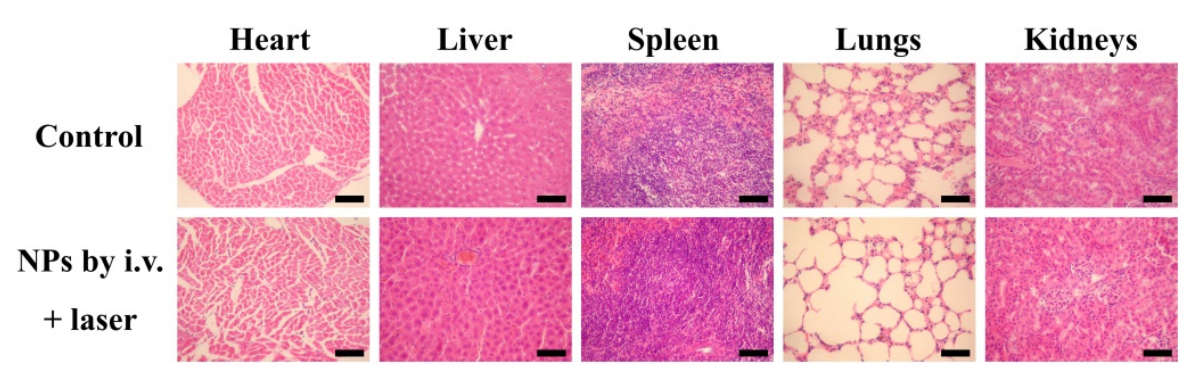

Figure A3. S14. H\&E stained splanchnic slices after 16 days of treatment. The control group is age-matched healthy mice. The NPs by i.v. + laser group is thermo-chemotherapy group in our experiments. The scale bar is $100 \mu \mathrm{m}$.

\section{References}

1. Li SY, Liu SW, Wang L, Lin M, Ge R, Li X, Zhang X, Liu Y, Zhang LN, Sun HC, Zhang H, Yang B. Tumor Theranostics of Transition Metal Ions Loaded Polyaminopyrrole Nanoparticles. Nanotheranostics 2018; 2(3): 211-221. DOI: 10.7150/ntno.25119.

2. Liu SW, Wang L, Lin M, Wang DD, Song ZQ, Li SY, Ge R, Zhang X, Liu Y, Li ZM, Sun HC, Yang B, Zhang H. Cu(II)-Doped Polydopamine-Coated Gold Nanorods for Tumor Theranostics. ACS Appl. Mater. Interfaces. 2017; 9(51): 44293-44306. DOI: 10.1021/acsami.7b13643. 